\title{
Optimal Frequency Control of a Microgrid
}

\author{
G. Mallesham ${ }^{1}$, Krishna Degavath ${ }^{2}$
}

Received: $10^{\text {th }}$ December 2017, Accepted: 17th January 2018, Published: 28th February 2018

\begin{abstract}
The paper provides an introduction to the complex problem of controlling a Microgrid. After defining the various terms used throughout the paper, a specific problem involving the control of a linear system with a constant load is stated. Both classical methods like PI control and optimal control method of quadratic performance index were attempted for designing the controller. The former was used to determine the improvement in performance achieved by using state feedback. The latter was found to be significantly better, and satisfied all the requirements, even when the load varied randomly with time
\end{abstract}

Keywords: Microgrid, Modeling, Frequency control, Optimal control.

\section{Introduction}

This is the first of a series of the papers that the authors intend to write on the control of a smart grid. It represents the cooperative effort on the part of two researchers, who have considerable experience in two different areas i.e. power systems and control theory. It is motivated by the strong belief on the part of both authors that such cooperation between workers in the two fields will be needed to address many of the problems that will arise in the future in energy management. This paper, as well as future contributions, will be organized to help such a mixed audience. The papers will deal with multiple energy sources which cooperate to meet the energy demands of a Microgrid (defined later), while optimizing a chosen performance index [1]. The problem is open ended since, at every stage, a variety of choices are open to the designer. These include the primary and secondary sources in the system, the criteria by which they are introduced into the system, the qualitative characteristics desired of the overall system, and the realization of these responses using appropriate qualitative performance criteria with fixed or adaptive parameters, At every stage, the requirements of power system engineers and those of control theorists are taken into account. The latter includes stability, robustness and satisfactory performance under a variety of perturbations on the system.

It is a truism in control system design that the simplest controller that can achieve all the objectives is the one that is generally chosen. This is because the simplest controller is also quite often the most robust. In view of this, in the present and future papers the controller structures will successively be made more complex only when some criterion or criteria are not met. Further, rational explanations will be provided for any increase in complexity. The Since the authors expect these papers to be of interest to a mixed audience drawn from multiple disciplines, every effort will be made to explain the problems addressed in both qualitative and quantitative terms, so that they can appreciate the choices made. Also, since this paper is the first of the series, a proper terminology is included for the benefit of the reader.

\section{The Microgrid}

By a Microgrid we mean a small-scale power grid that can operate independently, or in conjunction with the main electric grid in the area in which it is located. Alternatively, any small-scale localized station with its own power resources, generation, and loads with defined boundaries, qualifies as a Microgrid [2].

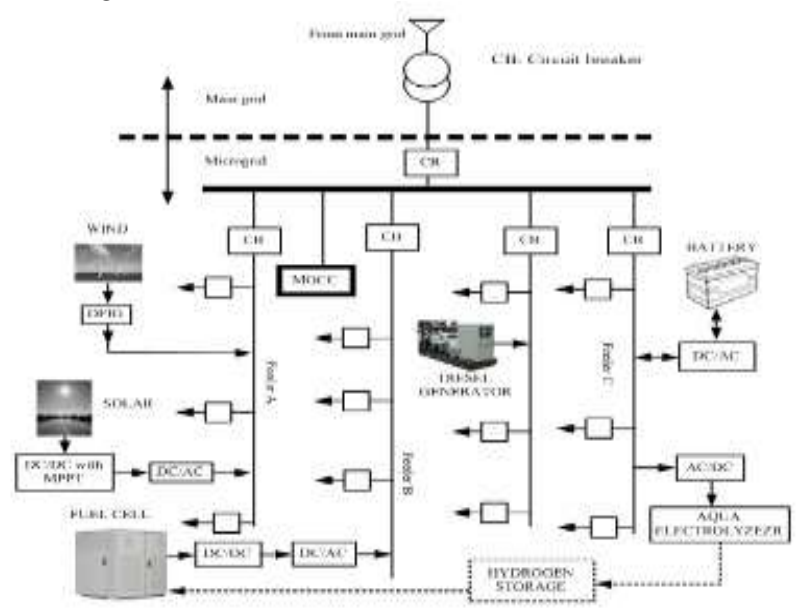

Fig. 1. The architecture of a Microgrid

The integration of small capacity power generating sources to an existing passive distribution system converts the latter into an active distribution network. In such a case they are said to operate in a grid connected mode. However, the occurrence of faults, voltage sags, or large frequency oscillations, may force them to be disconnected from the main grid, thereby forcing them to operate as an isolated Microgrid. The architecture of a Microgrid is given in Fig.1 [3].

The different types of small scale generation systems used in a Microgrid as shown in Fig. 1, can be categorized sources such as solar, wind, and biomass energy systems, while the latter includes diesel generators, fuel cells, aqua-electrolyzers, batteries, super capacitors, and flywheels [4]. 
The primary sources are categorized by the fact that they are not readily predictable i.e. they are not constant sources, but vary randomly with time. As a consequence, the power as well as the frequency of the Microgrid may fluctuate. To cope with such variations, the secondary sources are used. These, in turn, provide the power needed to compensate for the increase in load or decrease in power generation, thereby restoring equilibrium [5], [6], [7].

In the contemplated series of papers, the authors intend to consider a $500 \mathrm{~kW}$ Microgrid with all the primary and secondary sources referred to earlier. However, in this paper, the preliminary analysis will include only solar and wind as the primary power generators, and a diesel generator (DG) as a secondary source. The choice of the latter is based on the fact that the diesel generator has rotating parts and consequently plays a major role in the stability of the overall system. Fig.2 shows a diesel generator connected to a $500 \mathrm{~kW}$ Microgrid, integrated with solar power and wind power sources, each with a capacity of $250 \mathrm{~kW}$. This assures that there is adequate secondary power to compensate for fluctuations in the primary sources.

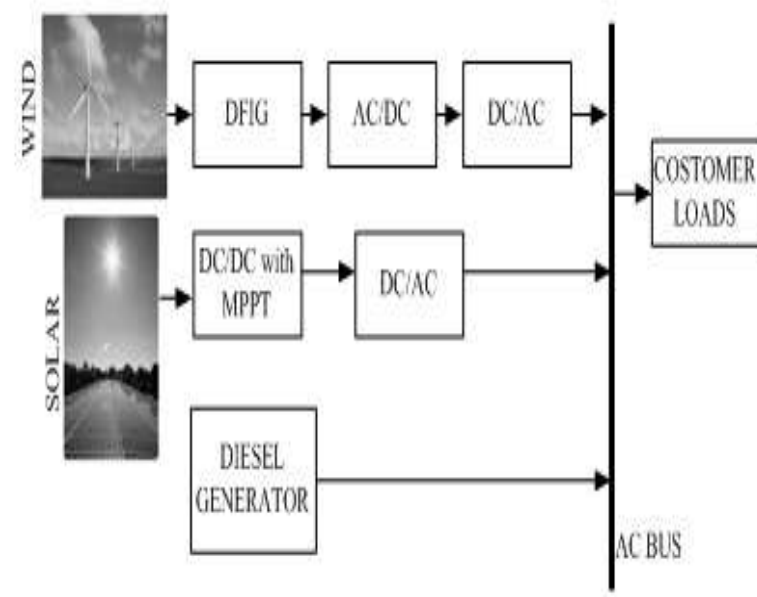

Fig.2. The block diagram of a Microgrid with diesel generator.

\section{Objectives}

In A block diagram representation of a Microgrid with two primary sources of capacity $250 \mathrm{~kW}$ each, is shown in Fig.3with a maximum expected load of $500 \mathrm{~kW}$. Due to random fluctuations in the primary sources, resulting in total power generation less than $500 \mathrm{~kW}$, a fixed load of $500 \mathrm{~kW}$ produces a power deficiency $\mathrm{P}(\mathrm{t})$, which is represented as the input to the Microgrid. The objective is to use secondary power sources to compensate for the deficiency dynamically. For reasons mentioned in the previous section, a Diesel generator is used in this paper as the secondary source. Taking all the dynamical components involved (to be discussed in this section) into account, the objective of the paper is to discuss and describe efficient procedures for designing controllers that will provide fast, stable, and robust compensation.

Comment 1: In the description given above, the load is constant at a maximum value of $500 \mathrm{~kW}$, while the two primary sources yield less power than is required by the load.

An alternative situation is one where the power available at any instant is greater than the demand at that instant. This leads to questions related to storage of the excess power. A more realistic scenario is one in which both situations are encountered as time evolves, due to varying loads. We shall consider such questions in future papers.

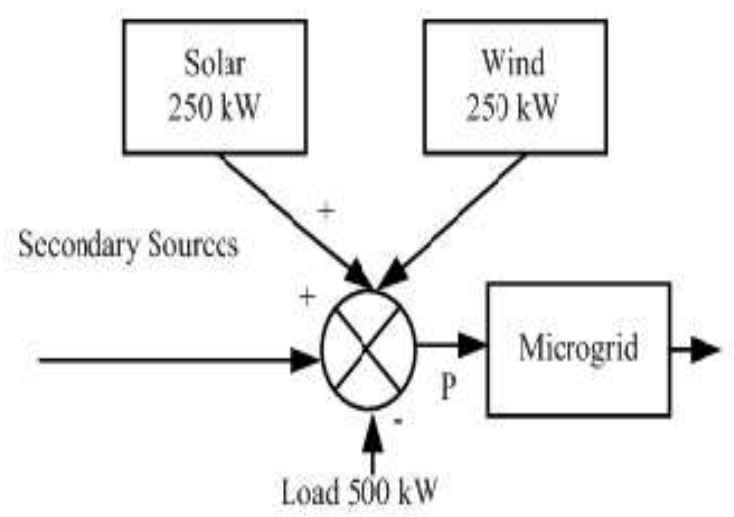

Fig.3. Block diagram representation of a microgrid with primary and secondary sources

Comment 2:While only two primary sources (solar and wind) and one secondary source (DG) are used in this paper, future papers will also consider the other sources described in the introduction.

\section{Statement of the Problem}

A block diagram of the overall system with the primarysources, load and the secondary source (DG) is shown in Fig.4.

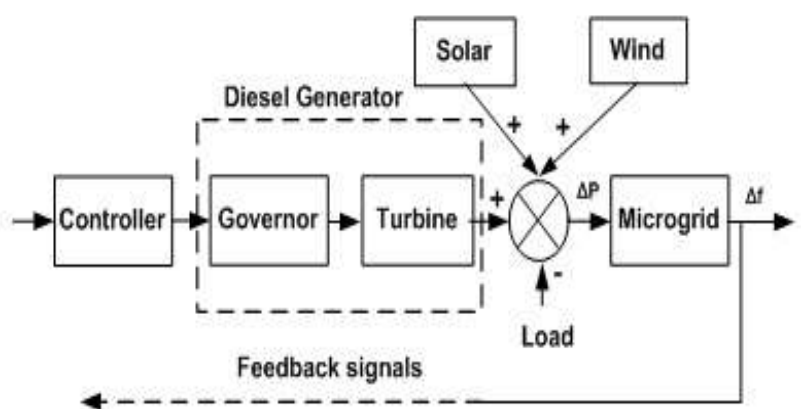

Fig. 4. A block diagram of the overall system with the primary sources, load and the secondary source (DG).

In The diesel generator consists of a governor and a 
turbine. A general controller is also indicated in Fig.4. Since many different control strategies will be described, this controller will have different inputs corresponding to each of them. The controller output is a single control signal to the diesel generator to provide the appropriate input shown in Fig.3 and Fig.4.

\section{The Linear Model}

While, in general, the dynamics of the various components may be nonlinear, we will be interested in a linearized representation around an operating (equilibrium) point. This implies that all the subsystems in the feedback loop will be represented by transfer functions, where the signals being discussed are small variations around the equilibrium state. As a result, the input to the Microgrid is represented by the variable $\Delta \mathrm{P}(\mathrm{t})$ (which is the variation of power around the nominal). Also, since the frequency of the Microgrid is an important variable, $\Delta \mathrm{f}(\mathrm{t})$, (the variation in frequency) is considered as the output of the linearized model of the Microgrid.

Assuming that the outputs of the governor and the turbine in the diesel generator, $\Delta \mathrm{P}(\mathrm{t})$ and $\Delta \mathrm{f}(\mathrm{t})$, and the outputs of the primary sources, as well as the load are measurable, the qualitative objective is to design a controller so that for random variations in the primary sources, the values of $\Delta \mathrm{P}(\mathrm{t})$ and $\Delta \mathrm{f}(\mathrm{t})$ achieve small values in a short interval of time and tend to zero. (i.e. fast response). Naturally, the stability of the overall system is a prerequisite for such behavior.

\section{Mathematical Models}

In the problem considered in detail in this paper the DG source and the Microgrid are assumed to have known transfer functions given in (1) and (2). The overall block diagram is shown in Fig.5 [7].

$$
\sum_{D G}: \frac{0.05}{s+0.05} \times \frac{0.5}{s+0.05}(\mathrm{DG} \text { source })
$$

$$
\sum_{M}: \frac{5}{s+0.06}
$$

(Microgrid)

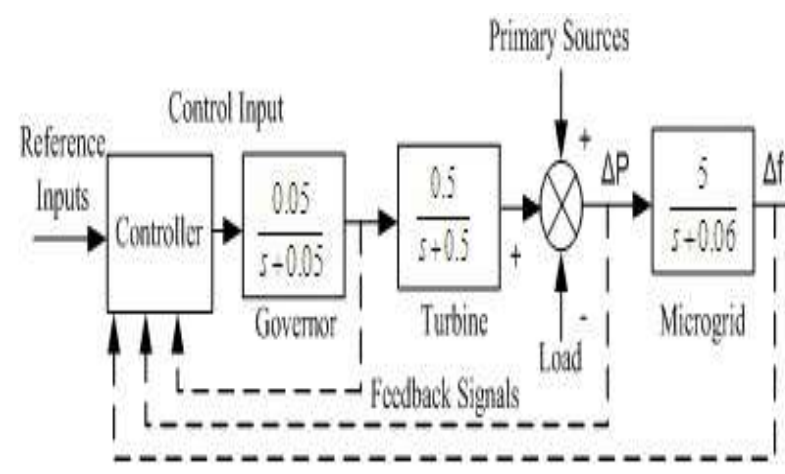

Fig.5. The overall system.

\section{The Problem}

The problem is to use various feedback signals and structures of the controller to assure stability, fast response, and robustness overall system. Towards this end, we shall propose various methods based on classical control theory, as well as those based on optimal control theory. Results from the former are generally simpler and may have advantages in some practical applications.

Under the power imbalance in the Microgrid due to changes in the load and the power output of primary sources, we have to maintain the frequency deviation of the Microgrid within the prescribed limits (say 0 . 05 ), from the nominal frequency of the system of 60 $\mathrm{Hz}$.

\section{The Mathematical Model}

The transfer function of the different subsystems are shown in Fig.5 and in Table I.

Table I: The Transfer Functions Of Subsytems

\begin{tabular}{|l|l|l|l|}
\hline & Input & Output & $\begin{array}{l}\text { Transfer } \\
\text { Function }\end{array}$ \\
\hline Governor & $\begin{array}{l}\text { Control } \\
\text { Input }\end{array}$ & $\begin{array}{l}\text { Steam } \\
\left(X_{3}(t)\right)\end{array}$ & $\frac{0.05}{s+0.05}$ \\
\hline Turbine & Steam & $\begin{array}{l}\text { Power } \\
\left(X_{2}(t)\right)\end{array}$ & $\frac{0.5}{s+0.5}$ \\
\hline Microgrid & $\begin{array}{l}\text { Power } \\
\begin{array}{l}\text { sources } \\
\text { Secondary } \\
\text { sources }\end{array}\end{array}$ & $\begin{array}{l}\text { Frquency } \\
\left(X_{1}(t)\right)\end{array}$ & $\frac{5}{s+0.06}$ \\
\hline
\end{tabular}

$$
\begin{aligned}
& \dot{x}_{1}=-0.06 x_{1}\left[x_{2}+\sum_{1}^{2} u_{1}-L\right] \\
& \dot{x}_{2}=-0.5 x_{2}+0.5 x_{3} \\
& \dot{x}_{3}=-0.05 x_{3}+0.05 u_{4}
\end{aligned}
$$

Where $x_{1}$ Microgrid Frequency $\Delta f, x_{2}=$ Output of the Turbine,

$\mathrm{X}=$ output of the governor.

$\mathrm{u}_{1}=$ Solar power in $\mathrm{kW}, \mathrm{u}_{2}=$ wind power in $\mathrm{kW}, \mathrm{u}_{3}$ $=$ Output of turbine in $\mathrm{kW}, \mathrm{u}_{4}=$ controller output.

It is assumed that all the state variables are accessible and that the power deficiency i.e. $\mathrm{P}$, can also be measured directly.

The state vector equation can be expressed as $\dot{x}=A x+b u$ where the matrix $\mathrm{A}$ and vector $\mathrm{b}$ are respectively

$$
A=\left[\begin{array}{ccc}
-0.06 & 5 & 0 \\
0 & -0.5 & 0.5 \\
0 & 0 & -0.05
\end{array}\right]
$$




$$
b=\left[\begin{array}{c}
0 \\
0 \\
0.05
\end{array}\right]
$$

It is seen that both the governor and the Microgrid have slow responses with time constants an order of magnitude larger than that of the turbine.

\section{Classical Control}

Proportional control (P) uses a single gain $\mathrm{K}_{\mathrm{P}}$ in the feedback path. In the present case the feedback signal can be either $\Delta \mathrm{P}(\mathrm{t})$ or the output of the Microgrid $\mathrm{x}_{1}(\mathrm{t})$ (frequency deviation $\Delta \mathrm{f}(\mathrm{t})$ ). Both signals were used in simulation studies, and it was decided that $\Delta \mathrm{P}(\mathrm{t})$ is to be preferred, due to the large time constant of the Microgrid [8].

Proportional and Derivative (PD), Proportional and Integral (PI) or Proportional, Integral and Derivative (PID) controllers can also be used on combinations of several signals. While such controllers are very simple and extremely well studied in both theory and practice, it is worth investigating how they perform in the system under consideration.

In this section several simulation studies are presented that have been carried out on different systems mentioned earlier, and comments are included on the results observed. Due to space limitations all the comments are necessarily brief. As stated earlier, we assume that the primary sources aswell as the load (i.e. $\mathrm{u}_{1} ; \mathrm{u}_{2}$, and $\mathrm{L}$ ) are constants, and require that $\Delta \mathrm{P}(\mathrm{t})$ and $\mathrm{x}_{1}(\mathrm{t})$ tend to zero asymptotically. Further, we require that their variations remain within specified bounds (mentioned earlier). In each of the simulations the Microgrid was simulated for $500 \mathrm{~s}$, and initially the load is assumed to be constant at $500 \mathrm{~kW}$. The solar and wind powers are $250 \mathrm{~kW}$ and together are capable of balancing the load. A sudden change in load demand of $5 \%$ is initiated at $10 \mathrm{Sec}$., which is compensated by the diesel generator. The response of the Microgrid is plotted corresponding to each method used.

\section{A. Proportional Control: $\left(K_{\mathbf{P}}\right)$}

As stated earlier, in the proportional controller the output

$\Delta \mathrm{P}(\mathrm{t})$ is feedback into the system as shown in Fig.6.

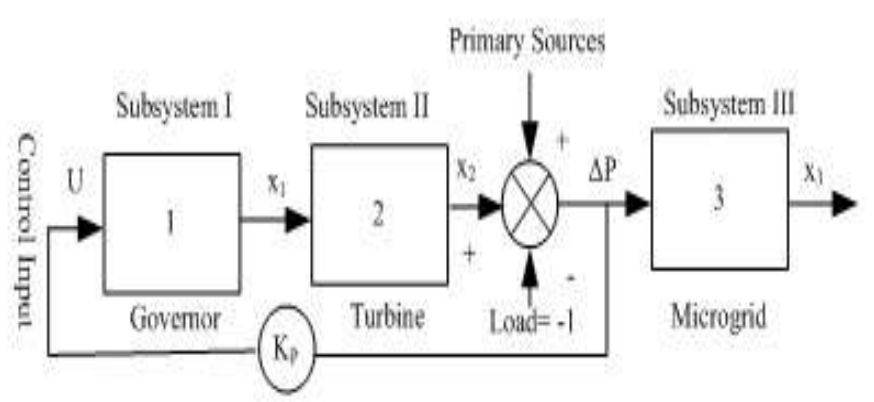

Fig.6. Block diagram of Microgrid with DG and Proportional controller.

The range of values of the gain for which the system is stable can be computed as 0:012 $<\mathrm{K}<0: 270$. The response obtained with $\mathrm{K}=0.1$ is shown in Fig.7. The amplitude of oscillations is $1.52 \mathrm{~Hz}$. Also, the steady state value of $\mathrm{x}_{3}$ is not zero $(0.5 \mathrm{~Hz})$ and the transients lie outside the desired frequency band. For the above reasons, the controller is found to be inadequate.

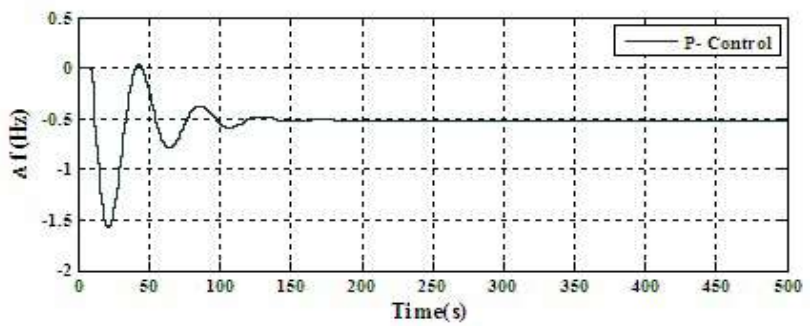

Fig. 7. Frequency response of the Microgrid with $P$ control.

Comment 3: A single parameter $K_{P}$ is inadequate to control the system satisfactorily.

\section{B. Proportional and Integral (PI) Control:}

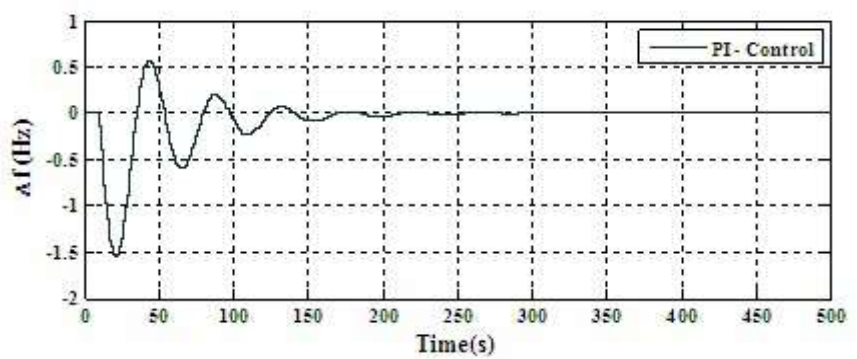

Fig. 8. Frequency response of the Microgrid with PI control.

From Fig.8, the frequency response of the Microgrid in the steady state is zero. However, the transient response makes the controller unsatisfactory. 


\section{Proportional Integral and Derivative (PID Control):}

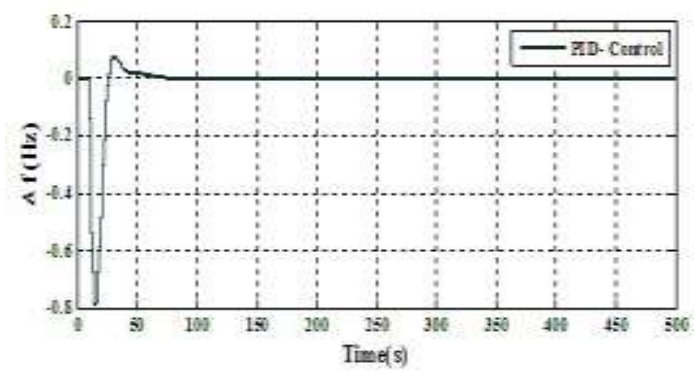

Fig. 9. Frequency response of the Microgrid with PID control.

From Fig.9, the Microgrid frequency response with PID is seen to be better than those shown in Figs.7 and Fig. 8 with $\mathrm{P}$ and PI controllers respectively. But the undershoot $(0.79 \mathrm{~Hz})$,is large. Fig.10 shows the responses of all the controllers for comparison purposes.

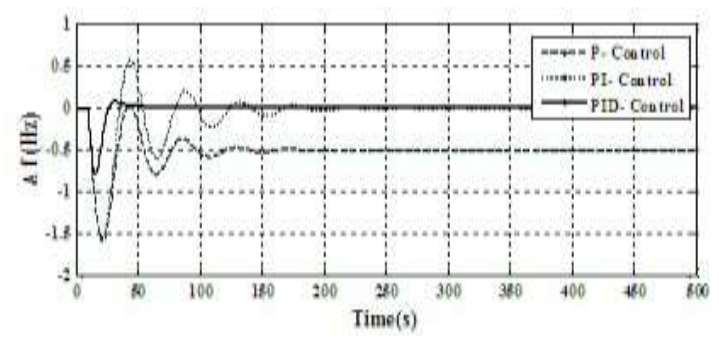

Fig.10. Comparison of Frequency response of the Microgrid with P, PI and PID control.

Comment 4: From preceding discussion it is clear that we have to explore other methods based on optimal control theory described in the next section.

\section{Optimal Quadratic Control}

Quadratic optimization of linear dynamical systems has become a standard tool for determining the optimal feedback parameters in applications involving linear dynamical systems [9], [10], [11], [12], [13]. However, when it is applied in practice in problems such as the one under investigation, certain precautions have to be taken and modifications made. Further, it is worth noting that simple control systems based on proportional, proportional and integral, and proportional integral and derivative control (P, PI, and PID) contain useful important information for choices to be made in the design of optimal controllers.

\section{The Basic Theory:}

If the dynamical system is described by the equation. It is to be noted here that (12) is valid only when there is a synchronous machine in the Microgrid. Therefore, the researchers should be careful in using (14) when they are simulating the Microgrid.

$$
\dot{x}=A x+b u \quad ; \quad x(O)=X_{0}
$$

The state regulator problem can be stated as the minimization of a performance index of the form

$$
J=\frac{1}{2} x^{T}[T] F x[T]+\frac{1}{2} \int_{0}^{T}\left[x^{T}[\tau] Q x[\tau]+u^{T} R u[\tau]\right] d \tau
$$

where $\mathrm{T}$ is the terminal time, $\mathrm{F}$ and $\mathrm{Q}$ are positive semi-definite matrices, and $\mathrm{R}$ is a positive definite matrix.

Comment 5: In a practical application, the matrices F, Q and R have to be suitably chosen. However, an explicit correlation between the choice of the parameters of these matrices and what the designer would consider as satisfactory performance" does not exist. Thus, ever since the beginning, a trial and error procedure has been used to choose the matrices. This involves solving the problem with a set of matrices, obtaining the solution as $\mathrm{u}(\mathrm{t})=\mathrm{G}^{1} \mathrm{X}(\mathrm{t})$, simulating the system with the matrix $\mathrm{G}(1)$ to generate the control input, and repeating the process by updating the matrices F, Q and R.

The optimization problem involves the solution of the nonlinear Riccatti equation.

$$
\begin{gathered}
\dot{K}+\stackrel{T}{A} K+K A-K B R^{-1} B^{T} K+Q=0 \\
K(T)=F
\end{gathered}
$$

and using the feedback matrix $G \in R^{n x r}$ to generate the control input $u(t)$ as

$$
u(t)=G x(t)=-R^{-1} B^{T} K x(t)
$$

In the problem of interest to us, the linear system is time invariant and the optimization is over an infinite horizon i.e. $T \rightarrow \infty$. This results in a constant $\mathrm{K}$ matrix and hence the feedback matrix $\mathrm{G}$, is also timeinvariant. Further $\mathrm{F}=0$, and the matrices $\mathrm{Q}$ and $\mathrm{R}$ are chosen to be diagonal, so that, desired changes in the variables of the system can be achieved by modifying the corresponding parameters in these matrices.

\section{Auxiliary State Variables}

To It was described earlier (section 5B) that an integrator has to be included in the feedback path to assure that $\mathrm{x}_{3}(\mathrm{t})$ (and hence $\left.\Delta \mathrm{P}(\mathrm{t})\right)$ tend to zero, even in the presence of a step input. The choice of this variables is a critical one, and it was decided that it should be $\Delta \mathrm{f}(\mathrm{t})$ as shown in Fig. 11 


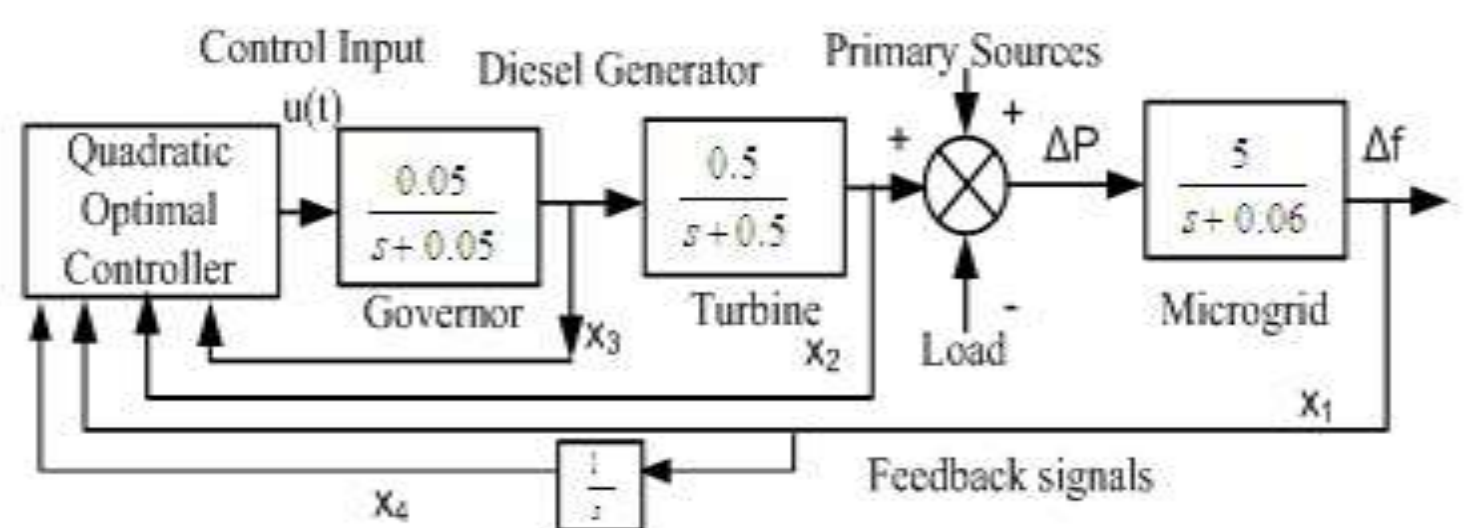

Fig. 11. The block diagram of Microgrid with Optimal Quadratic Controller

This increases the dimension of the control system by one, making $A \in R^{4 \times 4}$ and $b \in R^{4}$. The corresponding A matrix and vector are:

$A=\left[\begin{array}{cccc}-0.006 & 5 & 0 & 0 \\ 0 & -0.5 & 0.5 & 0 \\ 0 & 0 & -0.05 & 0 \\ 1 & 0 & 0 & 0\end{array}\right](13)$

And

$b=\left[\begin{array}{c}0 \\ 0 \\ 0.05 \\ 0\end{array}\right]$

The gain vector $\mathrm{G}$, was computed as

$\mathrm{G}=[104.6237331 .6793380 .5143]$

The state response of the system to a constant input (representing a 5\% variation) is shown in Fig.12.
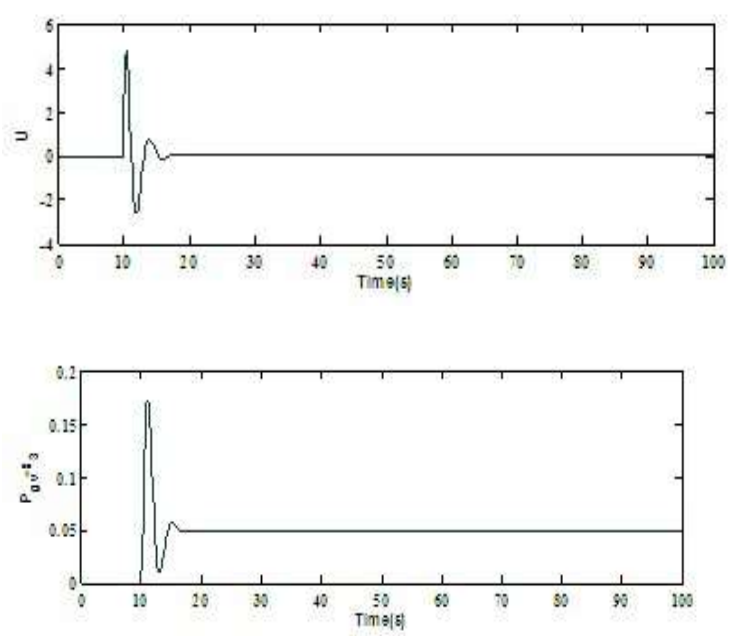
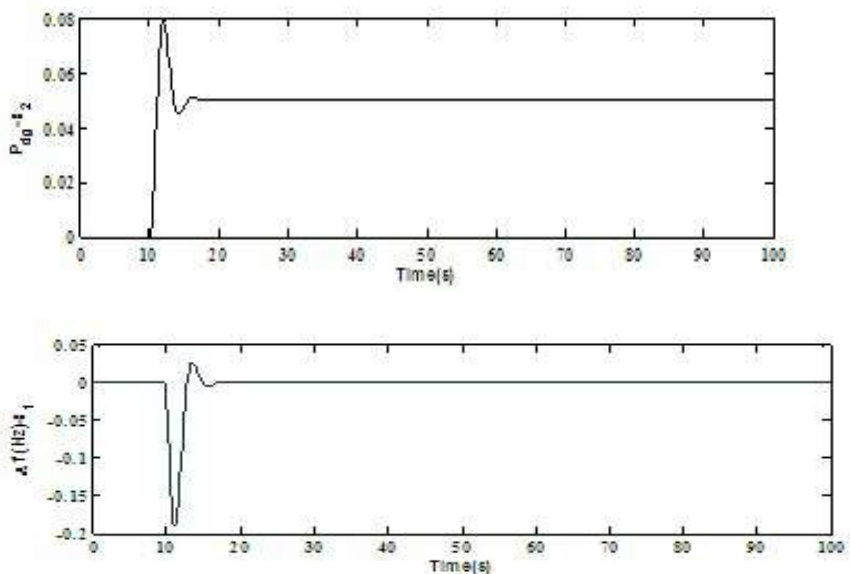

Fig. 12. (a), (b), (c) and (d) are Simulation responses of the Microgrid with Optimal Quadratic Controller.

From Fig.12, the frequency response of the Microgrid [14] is observed to tend to zero rapidly ( $25 \mathrm{sec}$.). The undershoot $(0.3 \mathrm{~Hz})$, is well within the frequency band for Microgrid stability. The responses using the different methods are shown bin Fig.13 for comparison purposes.

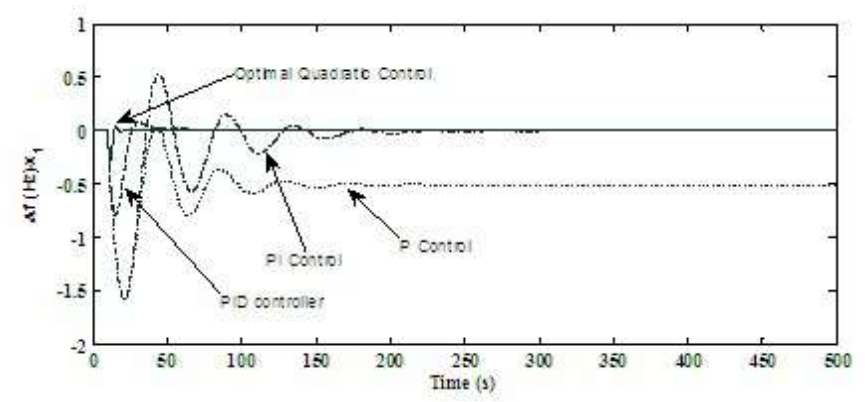

Fig. 13. Simulation responses of the Microgrid with different control methods. 
Comment 6: It is seen that performance of the optimalquadratic controller is significantly better than that of allthe classical controllers.

\section{Response To Random Variations In Solar And Wind}

As stated in the introduction, the true test of the performance of a controller is to be judged when the primary sources vary randomly with time [15]. This is shown in Fig.14. These random variations are in the primary source (wind power). There is also a sudden change in the wind power at $200 \mathrm{~s}$, and the corresponding response of the Microgrid with the optimal controller is shown in Fig.7.

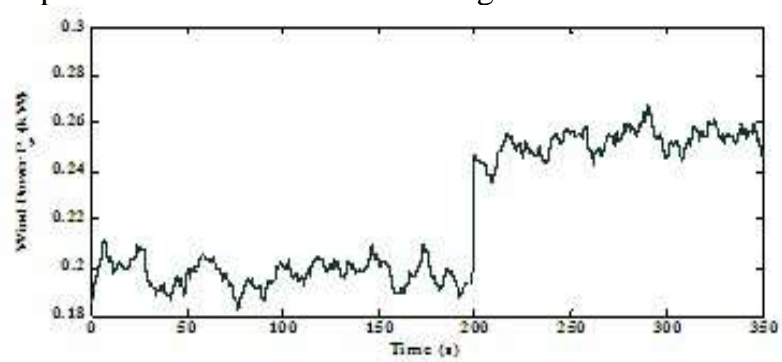

Fig. 14. Random variations in the Wind power in the Microgrid.
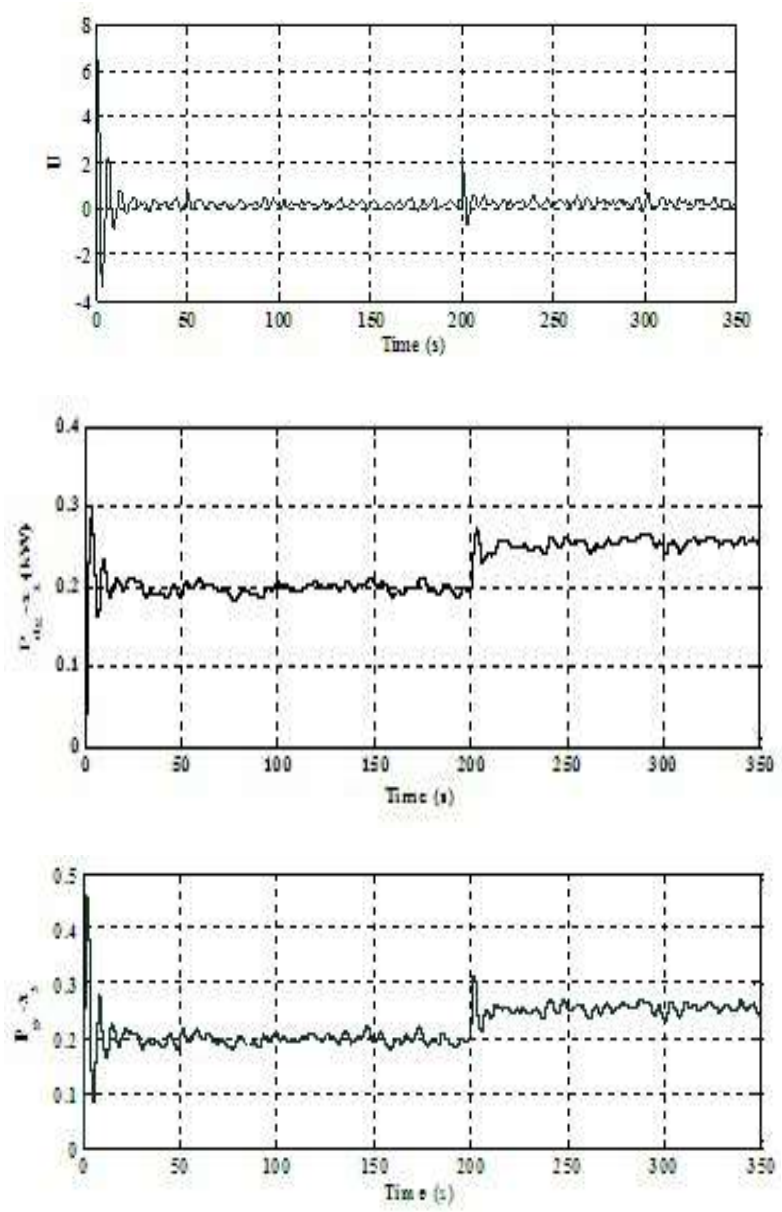

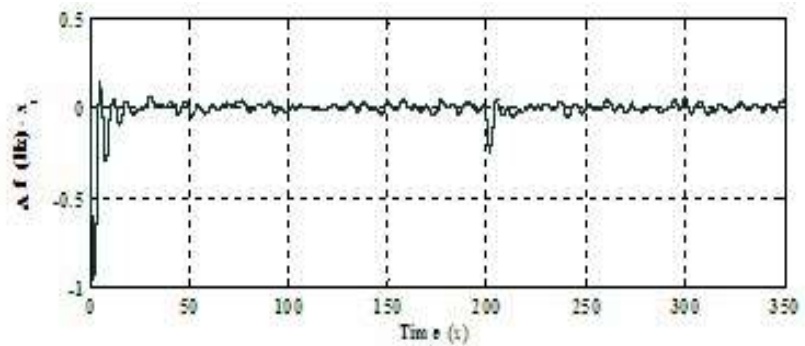

Fig.15.Simulation responses of the Microgrid with random variations in the primary sources.

\section{Future Work}

This paper introduced the main features of the problem of controlling the frequency variations in a Microgrid. Relevant terms were defined, the objectives were stated, and the control of a single linear time- invariant system, consisting of three subsystems with a single load was described using both classical methods and those based on optimal control. Future papers will deal with decentralized control, the relevance of concepts such as controllability and observability, the use of adaptive control in the presence of uncertainty, theoretical problems that arise when non-linearities are present and the design of nonlinear controllers using neural networks to cope with them.

\section{Conclusion}

Using both classical and modern methods based on optimal control, controllers were designed for a Microgrid. In each case the best parameters were obtained, and the corresponding responses of the overall system were simulated. The study of classical controllers is important to realize the best response that can be achieved using simple controllers. These responses are compared with that obtained using quadratic control, which requires significantly more information

\section{References}

[1] J. P. Lopes, C. Moreira, and A. Madureira, "Defining control strategies for Microgrids islanded operation," IEEE Transactions on power systems, vol. 21, no. 2, pp. 916-924, 2006.

[2] S. Chowdhury and P. Crossley, Microgrids and active distribution networks. The Institution of Engineering and Technology, 2009.

[3] S. Mishra, G. Mallesham, and P. C. Sekhar, "Biogeography based optimal state feedback controller for frequency regulation of a smart Microgrid," IEEE Transactions on Smart Grid, vol. 4, pp. 628-637, March 2013.

[4] M. Manas et al., "Renewable energy management through Microgridcentral controller design: An approach to integrate solar, wind and biomass with battery," Energy Reports, vol. 1, pp. 156-163, 2015. 
[5] M. A. Hassan and M. A. Abido, "Optimal design of Microgrids in autonomous and grid-connected modes using particle swarm optimization," IEEE Transactions on Power Electronics, vol. 26, pp. 755 769, March 2011.

[6] I. J. Balaguer, Q. Lei, S. Yang, U. Supatti, and F. Z. Peng, "Control forgrid-connected and intentional islanding operations of distributed power generation," IEEE Transactions on Industrial Electronics, vol. 58, pp.147-157, Jan 2011.

[7] S. Mishra, G. Mallesham, and A. N. Jha, "Design of controller andcommunication for frequency regulation of a smart Microgrid," IETRenewable Power Generation, vol. 6, pp. 248-258, July 2012.

[8] G. Mallesham, S. Mishra, and A. N. Jha, "Maiden application ofziegler-nichols method to agc of distributed generation system," in 2009IEEE/PES Power Systems Conference and Exposition, March 2009, pp. 1-7.

[9] I. Robandi, K. Nishimori, R. Nishimura, and N. Ishihara, "Optimalfeedback control design using genetic algorithm in multimachine powersystem," International journal of electrical power \& energy systems,vol. 23, pp. 263-271, 2001.

[10] O. Oral, L. C, Etin, and E. Uyar, "A novel method on selection of $\mathrm{q}$ andr matrices in the theory of optimal control." International Journal ofSystems Control, vol. 1, 2010.

[11] H. Smith and E. Davison, "Design of industrial regulators. Integral feedback and feedforward control," in Proceedings of the Institution of Electrical Engineers, vol. 119. IET, 1972, pp. 12101216.

[12] A. E. Bryson, Applied optimal control: optimization, estimation andcontrol. CRC Press, 1975.

[13] M. Johnson and M. Grimble, "Recent trends in linear optimal quadraticmultivariable control system design," in IEE Proceedings D (ControlTheory and Applications), vol. 134. IET, 1987, pp. 53-71.

[14] S. Murthy, S. Mishra, G. Mallesham, and P. Sekhar, "Voltage andfrequency control of wind diesel hybrid system with variable speed windturbine," in Power Electronics, Drives and Energy Systems (PEDES) \&2010 Power India, 2010 Joint International Conference on. IEEE,2010, pp. 16.

[15] L.-R. Chang-Chien, W.-T. Lin, and Y.-C. Yin, "Enhancing frequencyresponse control by dfigs in the high wind penetrated power systems,'IEEE transactions on power systems, vol. 26, pp. 710 718,2011 . 